\title{
A study of quiet eye's phenomenon in the shooting section of "laser run" of modern pentathlon
}

\author{
Andrea Chirico $^{1,2}$ | Dario Fegatelli $^{1}$ | Federica Galli ${ }^{1}$ | Luca Mallia $^{3} \mid$ \\ Fabio Alivernini ${ }^{4}$ | Susanna Cordone ${ }^{1}$ | Francesco Giancamilli ${ }^{1}$ | Stefano Pecci ${ }^{5}$ |

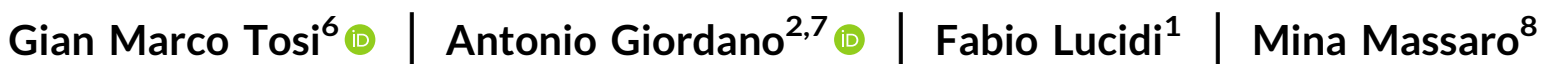

${ }^{1}$ Department of Psychology of Development and Socialization Processes, University "La Sapienza" of Rome, Rome, Italy

${ }^{2}$ Sbarro Institute for Cancer Research and Molecular Medicine, Center for Biotechnology, College of Science and Technology, Temple

University, Philadelphia, Pennsylvania

${ }^{3}$ Department of Movement, Human and Health Sciences, University of Rome "Foro Italico", Rome, Italy

${ }^{4}$ National Institute for the Evaluation of the Education System (INVALSI), Rome, Italy

${ }^{5}$ Italian Modern Penthatlon Federation, Sport Research Department

${ }^{6}$ Ophthalmology Unit of the Department of Medicine, Surgery and Neuroscience,

University of Siena, Siena, Italy

${ }^{7}$ Department of Medical Biotecnologies,

University of Siena, Siena, Italy

${ }^{8}$ Scheie Eye Institute, University of

Pennsylvania, Philadelphia, Pennsylvania

\section{Correspondence}

Antonio Giordano, Sbarro Institute for Cancer Research and Molecular Medicine, Center for Biotechnology, College of Science and Technology, Temple University, BioLife Science Bldg. Suite 431, 1900 N. 12th Street, 19122 Philadelphia, PA and Department of Medicine, Surgery and Neuroscience,

University of Siena, Siena, Italy.

Email: president@shro.org

Funding information

Pennsylvania Department of Health

\begin{abstract}
The aim of the study was to evaluate the effects of the Quiet eye (QE) phenomenon on performances during the shooting section of "Laser Run" of Modern Pentathlon, in two samples of athletes (novices and experts). The "Laser Run" consists of running and shooting activities. The study involved 18 experienced athletes of the Italian National Team of Modern Pentathlon (i.e., "elite" group) and 18 young and nonexpert athletes of a local Pentathlon club (i.e., "novice" group). Participants performed, in ecological conditions, five trials of four series of shootings (as it occurs in the real competitions), for a total of 20 series. During the shooting trials, athletes wore a mobile Eye Tracking System to record eye movements (saccades, blinks, and fixations). Key measures of the study were QE parameters (QE Duration [QED], Relative QED [RQED], and QE Onset), as well as the performance (accuracy and time to perform the event). The results revealed that both groups of athletes had a longer QED, RQED, and an earlier onset during their best shots than during the worse ones. Furthermore, differences between the groups showed that elite athletes had an earlier onset and a shorter QED than the novice group of athletes. These results provide insightful information about different cognitive and perceptual processes involved in Modern Pentathlon's athletes' performances at both the elite and nonelite level.
\end{abstract}

\section{KEYWORDS}

expertise, pentathlon, Quiet eye (QE), target shooting, visual behavior

\section{1 | INTRODUCTION}

The sporting arena provides an excellent "in vivo" lab in which to test theoretical assumptions related to motor performances. In an aiming sport, the ability to coordinate and program precise aiming movements and attention are crucial (Vickers, 1996a, 1996b). In these sports, the processing of critical visual information and the ability to self-regulate cognitive and emotional activity are keys to the successful execution of self-paced movement skills. (Tosi, et al., in press; Williams, Singer, \& Frehlich, 2002)

Between the variables related to the sports performances, gaze behavior, in particular, the "Quiet eye" (QE) phenomenon, was defined in 1996 by Vickers in a study on aiming task sports (Vickers, 1996a, 1996b). This phenomenon has been defined by the author as "the final fixation or tracking gaze that is located on a specific location or object (a relevant target) in the task environment within 
$3^{\circ}$ of the visual angle or less for a minimum of $100 \mathrm{~ms}$, before the execution of the critical phase of movement." Characteristics of the QE are: a specific location of the fixation; the start of the fixation, namely the onset, that occurs before the critical final phase of the movement; its duration; and the end of the fixation, namely offset that occurs when the gaze deviates off the location or object by more than $3^{\circ}$ for more than 100 ms (Vickers, 1996a, 1996b). In a study of Causer, Bennett, Holmes, Janelle, and Williams (2010), the authors compared QE in different specialties of clay target pidgeon with different timing. The duration of the QE has been parameterized as relative to the time spent by the athletes, labeled as Relative QE Duration (RQED). RQED was the duration of the phenomenon divided the time used to perform the action. This represents the percentage of the time that the athlete is engaged in the QE relative to the duration of the execution of the entire skill (Lebeau et al., 2016).

Over the past 20 years, several studies showed that QE has a significant relationship with the athlete's sports performance. Specifically, these studies took into account some features of this phenomenon provided in different sports disciplines, that is golf (Vickers, 1992), basket (De Oliveira, Huys, Oudejans, Van De Langenberg, \& Beek, 2007; Oudejans, Koedijker, Bleijendaal, \& Bakker, 2005; Vickers, 1996a, 1996b), billiard (Williams et al., 2002), rifle shooting (Janelle et al., 2000), clay target pidgeon (Causer et al., 2010; Causer, Holmes, \& Williams, 2011; Causer, Holmes, Smith, \& Williams, 2011), and biathlon (Vickers \& Williams, 2007). As evidenced by these studies and by several reviews on this topic, an earlier QE onset and a longer Quiet Eye Duration (QED) and RQED correlated with a higher performance and/or a higher level of the athletes' expertise (Fegatelli, Giancamilli, Mallia, Chirico, \& Lucidi, 2016; Lebeau et al., 2016; Rienhoff, Tirp, Strau, Baker, \& Schorer, 2016).

Despite the robustness of the empirically identified phenomenon and progress over the recent years (for an overview, see Gonzalez et al., 2015), the mechanisms underlying the QE effect are still not well understood. From a theoretical point of view, different hypotheses have been proposed to explain the relationship between $\mathrm{QE}$ and performance. One of the predominant hypotheses is "the programming hypothesis" (Horn, Alexander, Gardin, Sylvester, \& Okumura, 2012; Mann, Coombes, Mousseau, \& Janelle, 2011; Williams et al., 2002). In line with this hypothesis, the QE facilitates information processing, and its duration seems to reflect the time needed to program the motor behavior and to accurately tune the response. Thus, longer QEDs are thought to extend this critical motor preparation period, enhancing performance (Mann et al., 2011; Vickers, 2011). Williams et al. (2002), in a study on billiard players, reported longer QEDs with increased levels of task complexity, and therefore a reduction of QED when the time available for the task was experimentally reduced. Their findings support the programming hypothesis, in that longer QED corresponds to greater information processing demands for complex tasks, requiring longer programming times. Furthermore, according to the affordance hypothesis, different authors, manipulating the availability of visual information, demonstrated that QE has not only the function to preprogram the motor behavior (offline control) but also to act as a behavioral control (online control; De Oliveira et al., 2007; Oudejans, Van De Langenberg, \& Hutter, 2002). Vine, Lee, Walters-Symons, and Wilson, (2015) in their study were able to also calculate the proportion between the two different QE functions (offline vs. online control). From a neuro-behavioral perspective, some authors found that a QE duration might reflect two different purposes relative to internal movement plans (Corbetta, Patel, \& Shulman, 2008; Vine et al., 2015). These different purposes have been linked to a delicate tradeoff between two different streams of bio-visual information processing: top-down (dorsal attentional network [DAN]) and bottom-up (ventral attentional network [VAN]) control networks (Corbetta et al., 2008), both involved in target selection and computations for movement parameterization during the QE (Gonzalez et al., 2015). According to Corbetta, the first (DAN) is a goal-directed attentional system centered on the dorsal posterior parietal and frontal cortex and allows one to link relevant stimuli to response planning, whereas the second (VAN) is a stimulus-driven attentional system centered on the temporoparietal and ventral frontal cortex that intrudes with the previous during the detection of salient stimuli. Whereas the amygdala (involved in emotional regulation) and hippocampus (involved in recording memories) are enclosed in the VAN system, Vickers (2012) suggests that "when a long duration QE is maintained on an optimal location a mental buffer or barrier is created that prevents intruding thoughts or bad experiences arising in the hippocampus and amygdala from distracting attention and leading to higher levels of anxiety;" this can improve the performance. However, this explanation does not fully describe the positive facilitator effects of the QE or define the actual information that is being processed (Gonzalez et al., 2015). Furthermore, the notion that experts have longer QEDs reflecting prolonged attention and motor preparation time questions whether only open-loop programming mechanisms are active during this extended time (Vine, Lee, Moore, \& Wilson, 2013), which coherently, would need a major online control. The findings of longer duration of the QE in expert athletes have led some scientists to investigate it, in particular, labeling it as the "efficiency paradox" (Mann, Wright, \& Janelle, 2016). The paradox lays on the "controlled versus automatic" processes mainstream (e.g., Fitts \& Posner, 1967); consistent with this theory, the motor expertise is generally characterized by the automatization of the process underlying the motor performance, reported in the literature as a decrease in reaction times, processing demands and also in aiming task experiment (Lucidi, Grano, Barbaranelli, \& Violani, 2006; Maslovat, Hodges, Chua, \& Franks, 2011; McMorris \& Graydon, 2000), contrasting, then, with the "programming hypothesis." Consequently, Klostermann, Kredel, and Hossner (2014) proposed the inhibition hypothesis, with reference to Neumann and Deschepper (1992), an alternative explanation of the QE phenomenon that is still rooted in the cognitive domain relying on the selection-for-action mechanism (Allport, 1987; Cisek \& Kalaska, 2010; Neumann, 1996), suggesting the QE as a "shielding mechanism" to inhibit nonoptimal task solutions selecting the optimal 
movement to execute. In this sense, it can be hypothesized that the increasing number of alternative task solutions gathered over years of practice comes with increasing shielding demands that, in turn, leads to the prediction of longer QE durations for experts than for novices or near-experts (Klostermann \& Hossner, 2018).

Most of the studies evaluating QE were related to far aiming tasks in self-paced sports. In these sports, the time used to perform does not affect performance (i.e., archery, basketball free throw, golf putting, pistol and rifle shooting, and soccer penalty kicks). The athletes, in these tasks, can perform without time constraints. The best performance corresponds to the best score. A few studies investigated the $\mathrm{QE}$ phenomenon in shooting aiming tasks where there is a limited time to hit a target.

For example, Causer et al. (2010), in their studies, evaluated QE parameters in different specialties of clay target pigeon (trap, double trap, and skeet). In these specialties, time constraint depends on the speed of the target (the plate) established by the rules of the sport for each specialty. Temporal constraint depends on external factors and is common to all the athletes. In their studies, Causer, Holmes, Smith et al. (2011) showed how QE characteristics were different depending on the expertise of the group (elite athletes showed an earlier onset QE and a longer RQED compared with novice athletes) and anxiety conditions (high-anxiety conditions could lead to a later onset and a shorter QED than in low-anxiety ones). In these studies the authors found the same patterns of differences in QE parameters in relation to the accuracy: best shots were characterized by an earlier QE onset and a longer QED. Furthermore, in another study, a QE training procedure was evaluated for skeet specialty. Results showed an earlier QE onset, a longer QE, and a reduction of the velocity (peak velocity), in a group of athletes who received training compared to their colleagues in the control group.

Another example has been provided by a study of Vickers and Williams (2007) that evaluated the differences in QE parameters and accuracy at different pressure conditions (low-pressure vs. high pressure) and power output (percentage of maximum oxygen uptake), in a sample of 10 National Biathlon athletes. In this sports time becomes a significant element in the performance; in fact, the athletes try to hit the targets as quickly as possible, so they can start skiing to reach the finish line. The biathlon rules state that each missed shot at the range generally involves a penalty lap of $150 \mathrm{~m}$, stressing the importance of being accurate rather than fast during the execution of the task. Results from this study relating to the differences between QE parameters and performance showed that best shots were characterized by an earlier QE onset and a longer QED.

The aim of the current study is to investigate the QE phenomenon in two samples of athletes of different expertise (novices vs. experts), in a timed targeting sport: the shooting section of the "Laser Run."

"Laser Run" is the final trial of Modern Pentathlon; it consists of running and shooting activities. The goal of this sport is to reach the finish line before the other athletes. The shooting involves four series of $10 \mathrm{~m}$ pistol shootings in a range equipped with targets. Each of the four series of shootings is followed by running for $800 \mathrm{~m}$. Each series consists of hitting five targets with an unlimited number of shots in a maximum time of $50 \mathrm{~s}$ on a target with a valid zone of dimension $59.5 \mathrm{~mm}$ (score $\geq 7.3$ ). Thus, the athletes start to run immediately after the target has been hit correctly five times. The time taken during the shooting range is detected electronically: the time starts when the first shot hits the target (irrespective of whether it is higher or lower than a 7.3 score) and stops when the fifth correct shot (score $\geq 7.3$ ) hits the target. Scores range from 0 to 10.9; the latter corresponds to the perfect center of the target.

Thus, during "Laser Run," the best athlete will be the one who runs and shoots fastest. Paradoxically, during a competition an athlete could miss the target several times; however, he could finish the shooting task as first due to his rapidity of execution rather than his accuracy in shooting the target because there are no penalties for missed shots. In this sport, the athlete's performance depends on two different parameters: accuracy (score equal to or more than 7.3) and speed (time taken to complete the task). The performer can control the time at which the skill (shooting) is executed (self-paced skill), but he should try to be as fast as possible in the execution to exit the shooting range.

To date, there have been no attempts to examine visual search in such targeting tasks where the time is a crucial part of the performance, and no studies have looked at how these factors interact with the shots' accuracy and the expertise of the athletes.

The hypothesis of this study is that given the specific nature of the aiming task in the "Laser Run" the QE phenomenon will emerge as in literature in relation to shots' accuracy. With respect to the expertise group differences, there are no other studies evaluating these differences in this specific timed sports.

We expect that

a. Best shots will be related to longer QE and earlier onset than worse shots both in expert and in novice athletes.

b. Expert athletes, given the specificity of this sport, will be able to have a better performance using less time than their novice colleagues.

c. Therefore, given the specificity of the sport and in line with the above-mentioned literature (Williams et al., 2002), we expect that the elite athletes will be able to activate QE earlier (QE onset) than novice athletes; then they would significantly reduce the time of execution and consequently the QE duration.

\section{2 | MATERIALS AND METHOD}

\section{1 | Participants}

The study involved 36 athletes of the Italian Federation of Modern Pentathlon (FIPM), from two different agonistic levels. The first group was composed of 18 experienced athletes of the Italian National Team of Modern Pentathlon (i.e., "elite" group; 9 male; 9 female). These athletes were aged between 17 and 30 years (mean age $=24.3$; standard 
deviation $[S D]=4.76)$. The second group was composed of 18 nonexperts athletes of a local Pentathlon club (i.e., "novice" group; 10 male; 8 female). These athletes were aged between 14 and 19 years (mean age $=15.3$ years, $S D=1.84$ ). All athletes had normal or corrected-to-normal visual acuity. The athletes had a different dominant shooting eye: 31 athletes shot with only the right eye opened, 3 athletes shot with only the left eye opened, and 2 athletes shot with both eyes opened.

\section{2 | Gaze behavior measurement device}

Gaze was recorded using the SensoMotoric Instruments Eye Tracking Glasses (SMI Eye Tracking Glasses 2.0, SensoMotoric Instruments $\mathrm{GmbH}$, Teltow, Germany), a noninvasive video-based glasses-type eye tracker, and the SMI software "iView" (www.smivision.com). Gaze data were analyzed using an SMI-ETG laptop (Lenovo-X230) with the SMI software "BeGaze" (SMI; BeGaze, 3.3). The Eye Tracking Glasses and the SMI-ETG laptop were linked by a USB cable, properly set to allow participants to shoot freely. In particular, the apparatus consisting in a pair of glasses equipped with an external camera to record the athlete's visual field, and two internal cameras to record eye movements (saccades, blinks, and fixations). The portable computer was connected to the ETG through which it is possible to observe live what the eyewear was recording and then analyze the record and the data.

\section{3 | Procedures}

All participants were informed about the general purpose of our study, the eye tracker device was shown to each participant before the experiment, and he or she was then given the opportunity to ask questions regarding testing procedures. All participants provided written informed consent before taking part in the study.

The study was performed at the shooting range of the Italian National Olympic Committee in Rome, as ecological conditions.

Participants used their own personal laser handgun. All participants were required to follow the rules of the "combined event" discipline during the experiment, as agreed by the Union International of Modern Pentathlon: specifically, we asked the participants to perform five trials of four series of shootings for a total of 20 series.

Before starting the experiment, each participant was asked to warm up for at least 10 min without ETG. After the warm-up phase, the athletes were asked to wear the ETG, with the aid of the experimenter.

Given that the SMI ETG records the subject's gaze behavior of both eyes to enable monocular vision, we occluded the lens corresponding to the eye ordinarily kept closed by the athlete during the performance. This procedure does not hinder the data reporting by the instrument.

The calibration of the Eye Tracking Glasses was conducted using one reference point while participants were in their comfortable shooting stance. The accuracy of the calibration was checked periodically at the beginning of each shoot trial.

To familiarize with the procedure and the apparatus, participants were asked to perform a second warm-up phase with the ETG. The experiment started when each athlete was confident with the eye tracker glasses and the procedure.

\section{4 | Measures}

The study relied on the following key variables:

Action Time. The time, in milliseconds, occurring between a shot and the previous one. This index is not available for the first shot of each series.

Accuracy. The score of the shot was recorded as accuracy performance outcome; it ranges from " 0 " corresponding to a shot off of the target and 10.9 corresponding to the center of the target.

$Q E D$, corresponds to the time (ms) between the start of the $Q E$ (QE onset) and its end.

$R Q E D$, according to the literature on self-paced shooting aim sport, the RQED corresponds to the QE duration divided by the "Action Time." This represents the percentage of the time that the athlete is engaged in the quiet eye mechanism relative to the duration of execution of the entire skill (Lebeau et al., 2016).

Onset. The time from the start of the action (previous shot) and the start of the QE mechanism.

\section{5 | Statistical analysis}

According to procedures used in literature (e.g., Causer et al., 2010) we selected the 25 "best" shots and the 25 "worst" shots for each athlete considering the shots' accuracy (score). The key variables of the current study were calculated as the mean of those best and worst shots.

A series $2 \times 2$ mixed analysis of variance (ANOVA) was performed on the following measures: the Action Time, the score, and the QE parameters (QED, RQED, QE onset) using SPSS (version 25.0 SPSS Inc.). ANOVAs considered as independent variables: a "within subject" factor SCORE ("best vs. worst"), and a "between subjects" factor EXPERTISE ("elite" vs. "novice" athletes).

\section{3 | RESULTS}

\section{1 | Athletes' action time}

As reported in Table 1, significant differences emerged overall between the two groups $\left(F_{(1,34)}=73.57 ; p<0.001\right.$; partial eta squared $=0.684$ ); overall, "elite" athletes performed their shots faster (mean $=2.84)$ than the novice athletes (mean $=4.388$ ). No significant differences emerged, instead, between best and worst shots $\left(F_{(1,34)}=0.31 ; p=0.582\right.$; partial eta squared $\left.=0.009\right)$ and for the interaction between the factors considered in the analysis $\left(F_{(1,34)}=0.27 ; p=0.609\right.$; partial eta squared $\left.=0.008\right)$.

\section{2 | Athletes' performance}

As reported in Table 2, overall, elite athletes showed a significantly $\left(F_{(1,34)}=47.376 ; \quad p<0.001\right.$; partial eta squared $\left.=0.582\right)$ better 
TABLE 1 Action time across the expertise of the athletes and shots accuracy

\begin{tabular}{|c|c|c|c|c|}
\hline \multirow[b]{2}{*}{$\begin{array}{l}\text { Dependent } \\
\text { variable }\end{array}$} & \multicolumn{2}{|c|}{$\begin{array}{l}\text { Independent } \\
\text { variables }\end{array}$} & \multirow[b]{2}{*}{$\begin{array}{l}\text { Mean } \\
\text { time (ms) }\end{array}$} & \multirow[b]{2}{*}{$S D$} \\
\hline & Expertise & $\begin{array}{l}\text { Shots } \\
\text { accuracy }\end{array}$ & & \\
\hline \multirow[t]{4}{*}{ Action time } & \multirow[t]{2}{*}{ Elite } & Best & 2.840 & 0.240 \\
\hline & & Worst & 2.838 & 0.259 \\
\hline & \multirow[t]{2}{*}{ Novice } & Best & 4.723 & 0.951 \\
\hline & & Worst & 4.678 & 0.852 \\
\hline
\end{tabular}

Note. SD: standard deviation.

performances (mean score $=8.43$ ) than their novice colleagues (mean score $=7.24$ ). Independently of their expertise, the athletes reported a significantly better score in their best shots than in worst ones $\left(F_{(1,34)}=658.229, p<0.001\right.$; partial eta squared $\left.=0.951\right)$. Furthermore, the results showed a significant interaction effect comparing the two groups of athletes in their performance across best and worst shots $(F=48.156, p<0.001$; partial eta squared $=0.582)$. Overall, the elite athletes showed an accuracy rate of $76 \%$ (only $24 \%$ of all shots were lower than 7.3 ), whereas novice athletes had an accuracy rate of $52 \%$ ( $48 \%$ of all shots were lower than 7.3 ; Table 3 ).

\section{3 | QE parameters}

With respect QE duration, overall elite athletes showed a significant $\left(F_{(1,34)}=9.542 ; p=0.004\right.$; partial eta squared $\left.=0.219\right)$ shorter duration of their QE (mean time $=827.825 \mathrm{~ms}$ ) than novice athletes (mean time $=1655.901$ ).

Furthermore, independently of their expertise, the athletes showed significantly longer QED $\left(F_{(1,34)}=4.670 ; p=0.038\right.$; partial eta squared $=0.121$ ) in their best shots (mean $=1276.138 \mathrm{~ms}$ ) than in their worst shots (mean $=1207.588 \mathrm{~ms}$ ). No significant effect, instead, emerged for the interaction between the two factors considered $\left(F_{(1,34)}=0.139 ; p=0.712\right.$; partial eta squared $\left.=0.004\right)$.

With respect to the relative QED, the two groups showed no significant differences $\left(F_{(1,34)}=1.545 ; p=0.222\right.$; partial eta squared $=$ 0.043). However, the result showed that, overall, the athletes had

TABLE 2 Performance across the level of the athletes and shot accuracy

\begin{tabular}{lllll} 
& \multicolumn{2}{l}{$\begin{array}{l}\text { Independent } \\
\text { variables }\end{array}$} & & \\
\cline { 2 - 3 } $\begin{array}{l}\text { Dependent } \\
\text { variable }\end{array}$ & Expertise & $\begin{array}{l}\text { Shots } \\
\text { accuracy }\end{array}$ & Mean & \\
score & SD \\
Performance & Elite & Best & 9.835 & 0.146 \\
& & Worst & 7.033 & 0.558 \\
& Novice & Best & 9.676 & 0.156 \\
& & Worst & 4.796 & 1.241 \\
\hline
\end{tabular}

Note. SD: standard deviation.
TABLE 3 Quiet eye parameters across the level of the athletes and shots accuracy

\begin{tabular}{|c|c|c|c|c|}
\hline \multirow[b]{2}{*}{$\begin{array}{l}\text { Dependent } \\
\text { variable }\end{array}$} & \multicolumn{2}{|c|}{$\begin{array}{l}\text { Independent } \\
\text { variables }\end{array}$} & \multirow[b]{2}{*}{ Mean } & \multirow[b]{2}{*}{$S D$} \\
\hline & Expertise & $\begin{array}{l}\text { Shots } \\
\text { accuracy }\end{array}$ & & \\
\hline \multirow[t]{4}{*}{ QE duration } & \multirow[t]{2}{*}{ Elite } & Best & $856.197 \mathrm{~ms}$ & 334.894 \\
\hline & & Worst & $799.453 \mathrm{~ms}$ & 302.324 \\
\hline & \multirow[t]{2}{*}{ Novice } & Best & $1696.079 \mathrm{~ms}$ & 1098.432 \\
\hline & & Worst & $1615.722 \mathrm{~ms}$ & 1101.452 \\
\hline \multirow{4}{*}{$\begin{array}{l}\text { Relative QE } \\
\text { duration }\end{array}$} & \multirow[t]{2}{*}{ Elite } & Best & 0.300 & 0.121 \\
\hline & & Worst & 0.282 & 0.112 \\
\hline & \multirow[t]{2}{*}{ Novice } & Best & 0.377 & 0.237 \\
\hline & & Worst & 0.358 & 0.237 \\
\hline \multirow[t]{4}{*}{ QE onset } & \multirow[t]{2}{*}{ Elite } & Best & $2174.844 \mathrm{~ms}$ & 376.356 \\
\hline & & Worst & $2190.006 \mathrm{~ms}$ & 360.711 \\
\hline & \multirow[t]{2}{*}{ Novice } & Best & $3256.859 \mathrm{~ms}$ & 1135.787 \\
\hline & & Worst & $3397.521 \mathrm{~ms}$ & 1241.288 \\
\hline
\end{tabular}

significantly $\left(F_{(1,34)}=4.738 ; p=0.037\right.$; partial eta squared $\left.=0.122\right)$ longer relative QED in their best shots (mean $=0.339$ ) than in their worst shots (mean $=0.320$ ). Even for this variable, no significant effect for interaction emerged $\left(F_{(1,34)}=0.002 ; p=0.962\right.$; partial eta squared $=0.000$ )

Finally, with respect QE onset, the elite athletes, overall, reported a significantly $\left(F_{(1,34)}=15.470 ; p<0.000\right.$; partial eta squared $\left.=0.313\right)$ earlier onset $($ mean $=2182.425)$ than novice athletes (mean = 3327.190). Furthermore, overall, athletes, independent of their expertise, reported a significantly $\left(F_{(1,34)}=4.121 ; p=0.050\right.$; partial eta squared $=0.108$ ) earlier onset in their best shots (mean = $2715.851 \mathrm{~ms}$ ) than in their worst shots (mean $=2793.763 \mathrm{~ms}$ ). No significant effect for interaction, instead, emerged $\left(F_{(1,34)}=2.673\right.$; $p=0.111$; partial eta squared $=0.073$ ).

\section{4 | DISCUSSION}

The purpose of this study was to investigate the QE phenomenon during the "Laser Run" in two samples of athletes with different levels of expertise (novice and elite). The study can be considered the first in the literature that evaluates the QE phenomenon in a sport in which the athlete has to find an optimum trade-off between accuracy and time of execution to reach the best performance. In fact, during "Laser Run" competition, the best athletes will be the ones who run and shoot faster. For this reason, unlike other targeting sports, the goal of the shooting task is to be sufficiently accurate in hitting the target five times with a minimum score of 7.3 in the fastest time possible. So, apart from being able to accurately shots five times the target with a score higher than 7.3, it is advantageous for one to minimize the time spent for the execution 
of the action. Paradoxically during a competition, an athlete could miss the target several times; however, he could finish the shooting task before an athlete who decides to shoot only five perfect shots, without missing.

Overall, the data resulted from the evaluation of how $\mathrm{QE}$ parameters are related to the performance (best vs. worst), regardless of the expertise levels, found the same QE pattern of theexisting literature, confirming a significant relationship between the $\mathrm{QE}$ parameters (QED, RQED, and QE onset) and accuracy (Fegatelli et al., 2016; Gonzalez et al., 2015; Lebeau et al., 2016). Specifically, the data show that best shots are characterized by a longer QED, RQED, and an earlier QE onset than the worst shots.

As hypothesized, significant differences emerged by comparing the two samples in terms of the time of execution and accuracy. Elite athletes, in fact, performed their tasks better than their novice colleagues. Specifically, differences in terms of time of execution showed that the elite athletes performed their task with a mean time approximately $40 \%$ lower than the novice athletes. Furthermore, in terms of accuracy, the elite athletes showed a better accuracy than the novice one. Elite athletes showed a mean score of 8.4 (SD 0.33) compared with the 7.2 (SD 0.65) scored by novice athletes, suggesting that task difficulty for an elite athlete is quite low.

To evaluate how the QE parameters will differentiate the athletes, the two samples were then compared taking into account their level of expertise (elite vs. novice) and their level performances (best shots vs. worst shots) on the QE parameters (QED, RQED, and QE onset). Results showed a main effect of the expertise on the QED and QE onset variables. Overall, the elite athletes showed a shorter QED (mean time $=827.825 \mathrm{~ms}$ ) than the novice athletes (mean time $=1655.901 \mathrm{~ms}$ ), but at the same time, as expected, they started their quiet eye significantly earlier $($ mean $=2182.425)$ than the novice sample (mean $=$ 3327.190). Nonsignificant differences emerged between the two groups considering RQED $\left(F_{(1,34)}=1.545 ; p=0.222\right.$; partial eta squared $\left.=0.043\right)$.

To date, there have been no studies that examined visual search in such targeting tasks and evaluating how these factors interact with expertise and performance. Interesting data from our study showed elite athletes having a significantly shorter QED than the novice group; this result could be inferentially deducted from two orders of reasons. First, the differences between the two groups of athletes in terms of time spent in performing the action are relevant and could account also for the differences of their QED. In our study the time constraints are subjective and related to the expertise of the athletes who, during the years, trained their motor behavior in function of the specific task that involves time and accuracy together, with a specific emphasis on the time, and a task demand that was simple in terms of accuracy (not the best accuracy ever). This result is in line with the literature (e.g., Williams et al., 2002), in which results showed that reducing the availability of time to perform an aiming task, consequently, a reduction of the QED emerges.

Second, comparing the two group in terms of performances, it is interesting to underline that elite athletes' mean score (8.4) is quite higher than the demands of the task (7.3) compared with novice ones, performing instead, on average less than the minimum task required (7.2). Therefore, to account for this difference, an overall analysis of all the shots of the two groups of athletes showed that the elite athletes have an accuracy rate of $85 \%$ (only $15 \%$ of all shots were lower than 7.3) whereas novice athletes had an accuracy rate of $60 \%$ (40\% of all shots were lower than 7.3). This finding indicates that given the tradeoff needed for this sport, balancing accuracy and action time, the novice athletes set their priority towards accuracy to reach a complex shooting task, which was not difficult for the expert group of athletes. This result, therefore, could support the "programming hypothesis," in that longer QEDs correspond to greater information processing demands for complex tasks, requiring longer programming times, given the time availability. Williams et al. (2002) found shorter QE duration in billiard players who dealt with lower task demands than a higher difficulty task.

Fitts' law (Fitts, 1954) can provide useful information about the cognitive mechanism that could explain the trade-off between the time of execution (speed) and accuracy during this shooting task: this law, indeed, connotes an inverse relationship between the accuracy of a movement and the speed with which it can be performed. So, the elite athletes who showed a higher degree of accuracy compared with novice athletes, might decide to be less accurate, increasing the speed of movement to improve their performance in the task. Thus, the expertise of the athlete could allow more experienced athletes to execute the movement faster but still maintaining an adequate level of accuracy for this task, thus reducing the QE period, enough to reach the minimum score (minimum 7.3). Hence, it is reasonable that athletes with more expertise in a motor task where the time of execution is part of the performance will be more efficacious in balancing the trade-off between speed of movement and accuracy in the most functional way to obtain their best performance, thus reducing the time of execution and consequently the QED, anticipating their QE onset.

The study is the first to evaluate the "Laser Run" specialty; it, however, has some limitations. The results seem to provide coherent support to the statement "the quiet eye of elite performers is of an optimal duration, being neither too long nor too short, but ideal given the constraints of the task being performed" by Vickers, (2009), and could provide important information about some cognitive and perceptual processes involved in Modern Pentathlon athletes' performances at both the elite and non-elite level. Clearly, other studies need to confirm the inferences made in this study to confirm this results. Manipulations of the task difficulty and time available are needed to confirm the hypothesis related to the different duration of the QE between the two groups of athletes; subsequently, a bigger sample can be very useful to confirm the trends that did not reach statistical significance.

\section{CONFLICTS OF INTEREST}

The authors declare that they have no conflicts of interest.

\section{ACKNOWLEDGMENT}

Antonio Giordano and Andrea Chirico have been funded by the Pennsylvania Department of Health for Sbarro Health Research Organization (SHRO). 


\section{ORCID}

Gian Marco Tosi (D) http://orcid.org/0000-0001-9289-1717 Antonio Giordano (D) http://orcid.org/0000-0003-4353-5390

\section{REFERENCES}

Allport, A. (1987). Selection for action: Some behavioral and neurophysiological considerations of action. In Heuer, H., \& Sanders, A. F. (Eds.), Perspectives on Perception and Action (pp. 395-419). Hillsdale, NJ: Erlbaum.

Causer, J., Bennett, S. J., Holmes, P. S., Janelle, C. M., \& Williams, A. M. (2010). Quiet eye duration and gun motion in the elite shotgun shooting. Medicine and Science in Sports and Exercise, 42(8), 1599-1608. https://doi.org/10.1249/MSS.0b013e3181d1b059

Causer, J., Holmes, P. S., Smith, N. C., \& Williams, A. M. (2011). Anxiety, movement kinematics, and visual attention in elite-level performers. Emotion, 11(3), 595-602. https://doi.org/10.1037/a0023225

Causer, J., Holmes, P. S., \& Williams, A. M. (2011). Quiet eye training in a visuomotor control task. Medicine and Science in Sports and Exercise, 43(6), 1042-1049. https://doi.org/10.1249/MSS. Ob013e3182035de6

Cisek, P., \& Kalaska, J. F. (2010). Neural mechanisms for interacting with a world full of action choices. Annual Review of Neuroscience, 33(1), 269-298. https://doi.org/10.1146/annurev.neuro.051508. 135409

Corbetta, M., Patel, G., \& Shulman, G. L. (2008). The reorienting system of the human brain: From the environment to theory of mind. Neuron, 58 (3), 306-324. https://doi.org/10.1016/j.neuron.2008.04.017

De Oliveira, R. F., Huys, R., Oudejans, R. R. D., Van De Langenberg, R., \& Beek, P. J. (2007). Basketball jump shooting is controlled online by vision. Experimental Psychology, 54(3), 180-186. https://doi.org/10. 1027/1618-3169.54.3.180

Fegatelli, D., Giancamilli, F., Mallia, L., Chirico, A., \& Lucidi, F. (2016). The use of eye-tracking (ET) in targeting sports: A review of the studies on quiet eye (QE). In Pietro, G. D., Gallo, L., Howlett, R. J., \& Jain, L. C. (Eds.), Smart Innovation, Systems and Technologies (55, pp. 715-730). Springer. https://doi.org/10.1007/978-3-319-39345-2_64

Fitts, P. M. (1954). The information capacity of the human motor system in controlling the amplitude of movement. Journal of Experimental Psychology, 47(6), 381-391. https://doi.org/10.1037/h0055392

Fitts, P. M., \& Posner, M. I. (1967). Human performance, Belmont, CA: Brooks/Cole Pub. Co.

Gonzalez, C. C., Causer, J., Miall, R. C., Grey, M. J., Humphreys, G., \& Williams, A. M. (2015). Identifying the causal mechanisms of the quiet eye. European Journal of Sport Science, 1391, 74-84. https://doi.org/10. 1080/17461391.2015.1075595

Horn, R. R., Okumura, M. S., Alexander, M. G. F., Gardin, F. A., \& Sylvester, C. T. (2012). Quiet eye duration is responsive to the variability of practice and to the axis of target changes. Research Quarterly for Exercise and Sport, 83(2), 204-211. https://doi.org/10.1080/ 02701367.2012.10599851

Janelle, C. M., Hillman, C. H., Apparies, R. J., Murray, N. P., Meili, L., Fallon, E. A., \& Hatfield, B. D. (2000). Expertise differences in cortical activation and gaze behavior during rifle shooting. Journal of Sport and Exercise Psychology, 22(2), 167-182. https://doi.org/10.1123/jsep.22. 2.167

Klostermann, A., \& Hossner, E. J. (2018). The quiet eye and motor expertise: Explaining the "efficiency paradox.". Frontiers in Psychology, 9, https://doi.org/10.3389/fpsyg.2018.00104

Klostermann, A., Kredel, R., \& Hossner, E. J. (2014). On the interaction of attentional focus and gaze: The quiet eye inhibits focus-related performance decrements. Journal of Sport and Exercise Psychology, 36(4), 392-400. https://doi.org/10.1123/jsep.2013-0273
Lebeau, J. C., Liu, S., Sáenz-Moncaleano, C., Sanduvete-Chaves, S., Chacón-Moscoso, S., Becker, B. J., \& Tenenbaum, G. (2016). Quiet eye and performance in sport: A meta-analysis. Journal of Sport and Exercise Psychology, 38, 441-457. https://doi.org/10.1123/ jsep.2015-0123

Lucidi, F., Grano, C., Barbaranelli, C., \& Violani, C. (2006). Social-cognitive determinants of physical activity attendance in older adults. Journal of Aging and Physical Activity, 14(3), 344-359. https://doi.org/10.1123/ japa.14.3.344

Mann, D. T. Y., Coombes, S. A., Mousseau, M. B., \& Janelle, C. M. (2011). Quiet eye and the Bereitschaftspotential: Visuomotor mechanisms of expert motor performance. Cognitive processing, https://doi.org/10. 1007/s10339-011-0398-8

Mann, D. T. Y., Wright, A., \& Janelle, C. M. (2016). Quiet eye: The efficiency paradox-comment on Vickers. Current Issues in Sport Science, https://doi.org/10.15203/CISS

Maslovat, D., Hodges, N. J., Chua, R., \& Franks, I. M. (2011). Motor preparation and the effects of practice: Evidence from startle. Behavioral Neuroscience, 125, 226-240. https://doi.org/10.1037/ a0022567

McMorris, T., \& Graydon, J. (2000). The effect of incremental exercise on cognitive performance. International Journal of Sport Psychology, 31, 66-81. http://psycnet.apa.org/record/2000-08537-004

Neumann, O. (1996). Theories of attention, Handbook of Perception and Action (3, 389-446). Elsevier. https://doi.org/10.1016/S1874-5822(96) 80027-2

Neumann, E., \& Deschepper, B. G. (1992). An inhibition-based fan effect: Evidence for an active suppression mechanism in selective attention. Canadian Journal of Psychology/Revue Canadienne de Psychologie, 46(1), 1-40. https://doi.org/10.1037/h0084309

Oudejans, R. R. D., Koedijker, J. M., Bleijendaal, I., \& Bakker, F. C. (2005). The education of attention in aiming at a far target: Training visual control in basketball jump shooting. International Journal of Sport and Exercise Psychology, 3(2), 197-221. https://doi.org/10.1080/ 1612197X.2005.9671767

Oudejans, R. R. D., van de Langenberg, R. W., \& Hutter, R. I. (2002). Aiming at a far target under different viewing conditions: Visual control in basketball jump shooting. Human Movement Science, 21(4), 457-480. https://doi.org/10.1016/S0167-9457(02)00116-1

Rienhoff, R., Tirp, J., Strauß, B., Baker, J., \& Schorer, J. (2016). The 'Quiet eye' and motor performance: A systematic review based on newell's constraints-led model. Sports Medicine, 46(4), 589-603. https://doi. org/10.1007/s40279-015-0442-4

Tosi, G. M., Martone, G., Bacci, T., Tarantello, A., Baiocchi, S., Marigliani, S., ... Massaro-Giordano, G. (2019). Long term evaluation of corneal subbasal nerve recovery after PRK and influence of pars plana vitrectomy. Journal of Cellular Physiology, 234(5), 7459-7466.

Vickers, J. N. (1992). Gaze control in golf putting. Perception, 21(1991), 117-132. https://doi.org/10.1068/p210117

Vickers, J. N. (1996a). Control of visual attention during the basketball free throw. American Journal of Sports Medicine, 22(2), 342-354.

Vickers, J. N. (1996b). Visual control when aiming at a far target. Journal of Experimental Psychology: Human Perception and Performance, 22(2), 342-354. https://doi.org/10.1037/0096-1523.22.2.342

Vickers, J. N. (2009). Advances in coupling perception and action: The quiet eye as a bidirectional link between gaze, attention, and action. Progress in Brain Research, 174, 279-288. https://doi.org/10.1016/ S0079-6123(09)01322-3

Vickers, J. N. (2011). Mind over muscle: The role of gaze control, spatial cognition, and the quiet eye in motor expertise. Cognitive processing, 12, 219-222. https://doi.org/10.1007/s10339-0110411-2

Vickers, J. N. (2012). Neuroscience of the quiet eye in golf putting. International Journal of Golf Science, 1(1), 2-9. https://doi.org/10.1123/ ijgs.1.1.2 
Vickers, J. N., \& Williams, A. M. (2007). Performing under pressure: The effects of physiological arousal, cognitive anxiety, and gaze control in biathlon. Journal of Motor Behavior, 39(5), 381-394. https://doi.org/10. 3200/JMBR.39.5.381-394

Vine, S. J., Lee, D., Moore, L. J., \& Wilson, M. R. (2013). Quiet eye and choking: Online control breaks down at the point of performance failure. Medicine and Science in Sports and Exercise, 45, 1988-1994. https://doi.org/10.1249/MSS.0b013e31829406c7

Vine, S. J., Lee, D. H., Walters-Symons, R., \& Wilson, M. R. (2015). An occlusion paradigm to assess the importance of the timing of the quiet eye fixation. European Journal of Sport Science, 17(1), 85-92. https://doi.org/10.1080/17461391.2015. 1073363
Williams, A. M., Singer, R. N., \& Frehlich, S. G. (2002). Quiet eye duration, expertise, and task complexity in near and far aiming tasks. Journal of Motor Behavior, 34(2), 197-207. https://doi.org/10. 1080/00222890209601941

How to cite this article: Chirico A, Fegatelli D, Galli F, et al. A study of quiet eye's phenomenon in the shooting section of "laser run" of modern pentathlon. J Cell Physiol. 2019;234:

9247-9254. https://doi.org/10.1002/jcp.27604 